\title{
A METHOD OF DETERMINATION OF THE TEMPERATURE OF NUCLEI OF THE PLANETARY NEBULAE
}

\author{
G.S. KHROMOV \\ (Sternberg State Astronomical Institute, Moscow, U.S.S.R.)
}

I would like to make a few comments on the problem of the determination of the temperatures of nuclei of planetary nebulae.

It can be shown that in a high-excitation planetary nebula two independent Strömgren zones of heavy elements can be found. The first is a HeIII zone; the second corresponds to a zone of the luminescence of $\mathrm{Ne} v$ forbidden lines and originates due to combined absorption by the heavy ions $\mathrm{Ne} r \mathrm{v}, \mathrm{O} v$ and $\mathrm{N} v$. If so, a relatively independent method of the determination of the color temperature of nuclei can be suggested.

This method follows from a straightforward speculation, that in a stationary ionization zone the number of ionizations equals the number of recombinations and therefore, that the number of quanta in a corresponding interval of the stellar spectrum is proportional to the volume of this ionization zone.

If one knows the relative radii of some ionization zones in a given nebula one can calculate the relative intensities of the appropriate spectral intervals and then calculate the colour temperature of the star.

This method has been applied to 6 planetary nebulae with known angular diameters of the luminosity zones of $\mathrm{HeII}$ and $\mathrm{Nev}$, and the results are presented in Table 1. All of the colour temperatures lie in an interval from $100000^{\circ}$ to $200000^{\circ}$; their

Table 1

Temperatures of central stars

$\begin{array}{rccc}\text { Designation } & T_{\mathrm{c}} & T_{\mathrm{z}} & T_{\text {HeII }} \\ & & & 108000^{\circ} \\ \text { IC } 2165 & 140000^{\circ} & 49000^{\circ} & \\ \text { NGC } 2392 & 160000^{\circ} & 25000^{\circ} & 100000^{\circ} \\ \text { NGC } 2440 & 150000^{\circ} & 42000^{\circ} & 200000^{\circ} \\ \text { NGC } 6818 & 140000^{\circ} & 38000^{\circ} & \\ \text { NGC } 6886 & 160000^{\circ} & 52000^{\circ} & \\ \text { NGC } 7662 & 130000^{\circ} & 78000^{\circ} & \end{array}$

$T_{\mathrm{c}} \quad$ determinations by the author;

$T_{z} \quad$ Zanstra temperatures (Zanstra, 1960; Gurzadjan, 1962);

$T_{1 \mathrm{e}+\mathrm{I}}$ He II temperatures (Gurzadian, 1962; Aller, 1960; Zanstra, 1960).

Osterbrock and O'Dell (eds.), Planetary Nebulae, 330 331. C. I.A.U. 
individual scatter being moderate. The approximate temperatures obtained are much higher than the Zanstra temperatures (Zanstra, 1960; Gurzadian, 1962), but are of the same order of magnitudes as those determined by the HeII lines intensities (Gurzadian, 1962; Aller, 1960; Zanstra, 1960).

This result - independent of any distance or density estimates - probably proves that there is a significant ultraviolet excess in the spectra of nuclei of some planetary nebulae.

\section{References}

Aller, L. (1960) Bull. astr. Inst. Netherl., 15, 249.

Gurzadian, G. A. (1962) Planetary Nebulae, Moscow.

Zanstra, H. (1960) Bull. astr. Inst. Netherl., 15, 237.

\section{DISCUSSION}

Flower: I mentioned in my paper on Tuesday that I had made estimates of the combined opacities of ions such as NeIv, Ov, and Nv at about $100 \AA$. I find that the combined opacity rises to $0.5-1.0$ depending on the abundance values used. However, it is not clear that absorption of radiation by HeII can be neglected in the Nev ionization zone and if absorption by HeII is significant, it will change the central star temperature estimates of Dr. Khromov.

Khromov: The calculations mentioned were very qualitative, and there is a danger that something has been missed. But I would like to emphasize, that an independent luminosity zone of [Nev] still exists and I do not consider any other. It proves that there is an independent, corresponding luminosity zone. The contribution I have presented is a very brief account of a paper, which is in press in Mon. Not. R. astr. Soc. There are some facts in this original paper, which show that an additional source of opacity, if it still exists, does not affect the results significantly. (This paper has now appeared: Khromov, G.S. (1967) Mon. Not. R. astr. Soc., 137, 181.) 